\title{
ON STRONGLY PI-REGULAR RINGS OF STABLE RANGE ONE
}

\author{
HuA-PING Yu \\ Dedicated to Professor Victor P. Camillo \\ on the occasion of his fiftieth birthday
}

\begin{abstract}
An associative ring $R$ is said to have stable range one if for any $a, b \in R$ satisfying $a R+b R=R$, there exists $y \in R$ such that $a+b y$ is right (equivalently, left) invertible. Call a ring $R$ strongly $\pi$-regular if for every element $a \in R$ there exist a number $n$ (depending on $a$ ) and an element $x \in R$ such that $a^{n}=a^{n+1} x$. It is an open question whether all strongly $\pi$-regular rings have stable range one. The purpose of this note is to prove the following Theorem: If $R$ is a strongly $\pi$-regular ring with the property that all powers of every nilpotent von Neumann regular element are von Neumann regular in $R$, then $R$ has stable range one.
\end{abstract}

Let $R$ be an associative ring with identity. $R$ is said to have stable range one if for any $a, b \in R$ satisfying $a R+b R=R$, there exists $y \in R$ such that $a+b y$ is right (equivalently, left) invertible. Call a ring $R$ strongly $\pi$-regular if for every element $a \in R$ there exist a number $n$ (depending on $a$ ) and an element $x \in R$ such that $a^{n}=a^{n+1} x$. This is in fact a two-sided condition [2].

It is an open question whether all strongly $\pi$-regular rings have stable range one. Many special classes of strongly $\pi$-regular rings have been proved to have stable range one (see $[1,4,6,7])$. Goodearl and Menal [3] proved that strongly $\pi$-regular regular rings are unit-regular, hence have stable range one (Theorem 5.8, p.278). Here a ring $R$ is called unit-regular if for each element $x$ in $R$ there is a unit $u$ in $R$ such that $x u x=x$, that is, $x$ is a unit-regular element. We say also that an element $a$ of $R$ is (von Neumann) regular if $a=a x a$ for some element $x$ in $R$.

The main purpose of this note is to prove the following Theorem:

THEOREM 1. Let $R$ be a strongly $\pi$-regular ring. If all powers of every nilpotent regular element of $R$ are regular in $R$, then $R$ has stable range one.

To prove Theorem 1, we need the following characterizationby Goodearl and Menal $[3$, Theorem 6.1$]$ of stable range one for strongly $\pi$-regular rings .

Received 28th June, 1994

Example 4 was communicated to the author by Professor K.R. Goodearl.

Copyright Clearance Centre, Inc. Serial-fee code: 0004-9729/95 SA2.00+0.00. 
PROPOSITION 2. (Goodearl and Menal) For a strongly $\pi$-regular ring $R, R$ has stable range one if and only if every nilpotent regular element of the ring $e R e$ is unit-regular in $e R e$, where $e \in R$ is any idempotent of $R$.

The proof we are going to give is motivated by a proof of Goodearl and Menal [3, Theorem 5.8].

Proof of Theorem 1: Let $e^{2}=e \in R$ be any idempotent of $R$, and $x \in e R e$ be a nilpotent regular element of $e R e$, with $x y x=x, y \in e R e$. It suffices to prove, by Proposition 2, that $x$ is unit-regular in $e R e$.

Set $K_{i}=\operatorname{rann}_{e} \mathrm{Re}\left(x^{i}\right)$, the right annihilator of $x^{i}$ in $e R e$ for all $i=0,1,2, \cdots$.

ClaIM 1. There exists an integer $n \geqslant 1$ such that $x e R e+K_{n}=e R e$ and $x^{n} e R e \cap K_{1}=$ 0 .

This is clear, since we assume that $x^{n}=0$ for some $n \geqslant 1$.

ClaIm 2. $x e R e+K_{i}$ are direct summands of $e R e_{e R e}$ for all $i \geqslant 1$.

It is easy to check that an element of $e R e$ is regular in $e R e$ if and only if it is regular in $R$. So $x^{i}$ is regular in $e R e$ for all $i \geqslant 2$ by our assumption on $R$. We may assume $x^{i} y_{i} x^{i}=x^{i}$ for some $y_{i} \in e R e$ for $i \geqslant 2$. Then $K_{i}=\left(e-y_{i} x^{i}\right) e R e$. It is easy to check that

$$
x e R e+\left(e-y_{i} x^{i}\right) e R e=y_{i} x^{i} x e R e+\left(e-y_{i} x^{i}\right) e R e
$$

We check below that the element $y_{i} x^{i} x$ is actually von Neumann regular in $e R e$ :

$$
y_{i} x^{i} x \cdot y_{i+1} x^{i} \cdot y_{i} x^{i} x=y_{i} x^{i} x y_{i+1} x^{i} x=y_{i} x^{i} x
$$

Put $e_{i}=y_{i} x^{i} x y_{i+1} x^{i}$ and $f_{i}=e-y_{i} x^{i}$, then $e_{i} f_{i}=f_{i} e_{i}=0$. We see that $e_{i}$ and $f_{i}$ are orthogonal idempotents, hence $e_{i}+f_{i}$ is an idempotent. But $y_{i} x^{i} x e R e=e_{i} e R e$, so $x e R e+K_{i}=e_{i} e R e+f_{i} e R e=\left(e_{i}+f_{i}\right) e R e$ is a direct summand of $e R e_{e} e$.

Recall that we assume $x y x=x$, so $x e R e+K_{1}$ is a direct summand of $e R e_{e}$ e for the same reason.

ClaIm 3. $x^{i} e R e \cap K_{1}$ are direct summands of $e R e_{e}$ for all $i \geqslant 1$.

First, we show $x^{i} e R e \cap K_{1}=x^{i} K_{i+1}$. Since $x^{i} K_{i+1} \subset x^{i} e R e$ and $x^{i} K_{i+1} \subset K_{1}$, $x^{i} K_{i+1} \subset x^{i} e R e \cap K_{1}$; on the other hand, pick any $x^{i} r \in x^{i} e R e \cap K_{1}$, then $x x^{i} r=$ $x^{i+1} r=0$ so that $r \in K_{i+1}$; thus $x^{i} r \in x^{i} K_{i+1}$ and so $x^{i} e \operatorname{Re} \cap K_{1} \subset x^{i} K_{i+1}$.

Second, recall that we assume $x^{i} y_{i} x^{i}=x^{i}$, so that $K_{i+1}=\left(e-y_{i+1} x^{i+1}\right) e R e$, and we see that $x^{i}$ eRe $\cap K_{1}=x^{i} K_{i+1}=x^{i}\left(e-y_{i+1} x^{i+1}\right) e R e$. We check below that 
$x^{i}\left(e-y_{i+1} x^{i+1}\right)$ is von Neumann regular in $e R e$ :

$$
\begin{aligned}
x^{i}\left(e-y_{i+1} x^{i+1}\right) \cdot y_{i} \cdot x^{i}\left(e-y_{i+1} x^{i+1}\right) & =\left(x^{i}-x^{i} y_{i+1} x^{i+1}\right) y_{i} x^{i}\left(e-y_{i+1} x^{i+1}\right) \\
& =\left(e-x^{i} y_{i+1} x\right) x^{i} y_{i} x^{i}\left(e-y_{i+1} x^{i+1}\right) \\
& =\left(e-x^{i} y_{i+1} x\right) x^{i}\left(e-y_{i+1} x^{i+1}\right) \\
& =\left(x^{i}-x^{i} y_{i+1} x^{i+1}\right)\left(e-y_{i+1} x^{i+1}\right) \\
& =x^{i}\left(e-y_{i+1} x^{i+1}\right)\left(e-y_{i+1} x^{i+1}\right) \\
& =x^{i}\left(e-y_{i+1} x^{i+1}\right) .
\end{aligned}
$$

Therefore $x^{i} e R e \cap K_{1}=x^{i} K_{i+1}$ is a direct summand of $e R e_{e} R_{e}$.

Inasmuch as $x y x=x, x e R e \cap K_{1}=x K_{2}$ is a direct summand of $e R e_{e} e$.

ClAIM 4. (xeRe $\left.+K_{m}\right) / x e R e \cong K_{1} /\left(x^{m} e R e \cap K_{1}\right)$ for all $m$.

The right ideals of $e R e$ involved here are all direct summands of $e R e_{e R e}$ by Claim 2 and Claim 3 . We have the ascending and descending chains of direct summands

$$
\begin{gathered}
x e R e \subset^{\oplus} x e R e+K_{1} \subset^{\oplus} x e R e+K_{2} \subset^{\oplus} \cdots \subset^{\oplus} x e R e+K_{m} \\
K_{1}^{\oplus} \supset x e R e \cap K_{1}^{\oplus} \supset x^{2} e R e \cap K_{1}^{\oplus} \supset \cdots \oplus \supset x^{m} e R e \cap K_{1}
\end{gathered}
$$

which give us the decompositions

$$
\begin{aligned}
& \left(x e R e+K_{m}\right) / x e R e \cong \bigoplus_{i=0}^{m-1}\left(x e R e+K_{i+1}\right) /\left(x e R e+K_{i}\right) \\
& K_{1} /\left(x^{m} e \operatorname{Re} \cap K_{1}\right) \cong \bigoplus_{i=0}^{m-1}\left(x^{i} e \operatorname{Re} \cap K_{1}\right) /\left(x^{i+1} e \operatorname{Re} \cap K_{1}\right)
\end{aligned}
$$

so if we can show that

$$
\left(x e R e+K_{i+1}\right) /\left(x e R e+K_{i}\right) \cong\left(x^{i} e R e \cap K_{1}\right) /\left(x^{i+1} e \operatorname{Re} \cap K_{1}\right)
$$

for all $i$, we are done.

First we note that

$$
\begin{aligned}
\left(x e R e+K_{i+1}\right) /\left(x e R e+K_{i}\right) & =\left(x e R e+K_{i}+K_{i+1}\right) /\left(x e R e+K_{i}\right) \\
\cong K_{i+1} /\left[\left(x e R e+K_{i}\right) \cap K_{i+1}\right] & =K_{i+1} /\left[\left(x e R e \cap K_{i+1}\right)+K_{i}\right]
\end{aligned}
$$

As $x^{i} K_{i+1} \subseteq x^{i} e \operatorname{Re} \cap K_{1}$ and $x^{i}\left[\left(x e \operatorname{Re} \cap K_{i+1}\right)+K_{i}\right] \subseteq x^{i+1} e R e \cap K_{1}$, left multiplication by $x^{i}$ gives a module homomorphism

$$
f: K_{i+1} /\left[\left(x e \operatorname{Re} \cap K_{i+1}\right)+K_{i}\right] \rightarrow\left(x^{i} e \operatorname{Re} \cap K_{1}\right) /\left(x^{i+1} e \operatorname{Re} \cap K_{1}\right)
$$


$f$ is epic: Pick any $r \in x^{i} e \operatorname{Re} \cap K_{1}, r=x^{i} a$ for some $a \in e R e$. But, since $x^{i+1} a=$ $x r=0, a \in K_{i+1}$. So $f(a)=r$.

$f$ is monic: If $z \in K_{i+1}$ and $x^{i} z \in x^{i+1} e R e \cap K_{1}$, then we have $x^{i} z=x^{i+1} b$ for some $b \in e R e$ and $x^{i+2} b=x\left(x^{i} z\right)=0$, whence $x b \in K_{i+1} \cap x e R e$. Since $x^{i}(z-x b)=$ $0, z-x b \in K_{i}$, thus $z \in\left(x e R e \cap K_{i+1}\right)+K_{i}$, that is, $f$ is monic.

We have proved that $f$ is an isomorphism.

Claim 5. $x$ is unit-regular in eRe.

It follows from Claim 1 and Claim 4 that

$$
\left(x e R e+K_{n}\right) / x e R e=e R e / x e R e \cong K_{1} /\left(x^{n} e \operatorname{Re} \cap K_{1}\right)=K_{1} / 0=K_{1} .
$$

It is assumed that $x y x=x$, hence

$$
e R e=y x e R e \oplus K_{1}=x e R e \oplus(e-x y) e R e .
$$

So $K_{1} \cong(e-x y) e R e$. Denote this isomorphism by $\alpha$. Also, the restriction of the left multiplication by $x$ gives an isomorphism $\beta$ from yxeRe to xeRe. Define $u \in$ $e n d\left(e R e_{e R e}\right)=e R e$ to be the direct sum of $\alpha$ and $\beta^{-1}$. Then it is easy to check that $u$ is a unit in $e R e$ and $x u x=x$.

Yu [6] proved, among other things, that strongly $\pi$-regular rings whose idempotents are all central have stable range one. This result now can be easily deduced as a corollary of Theorem 1.

COROLlary 3. (Yu, [6]) Strongly $\pi$-regular rings whose idempotents are all central have stable range one.

Proof: It is known that a ring $R$ has stable range one if and only if $R / J(R)$ has stable range one, where $J(R)$ denotes the Jacobson radical of $R[5$, Theorem 2.2)]. Let $R$ be a strongly $\pi$-regular ring whose idempotents are all central. We need only to show that $R / J(R)$ has stable range one.

It is trivial to see that for a strongly $\pi$-regular ring $S$ with $J(S)=0$, all idempotents of $S$ are central if and only if $S$ contains no nonzero nilpotent element. Applying this equivalence to the factor ring $R / J(R)$, we see that $R / J(R)$ contains no nonzero nilpotent element, hence all powers of every nilpotent regular element are regular. So the conclusion follows from Theorem 1.

We conclude this note by giving an example, which shows that the converse of Theorem is false.

EXAMPLE. Let $R$ be the $2 \times 2$ matrix ring over $F[x] /\left(x^{2}\right)$, where $F$ is any field and $F[x]$ is the polynomial ring over $F$. 
Clearly, $R$ is a finite dimensional algebra, hence strongly $\pi$-regular. Of course, $R$ has stable range one. But not all the powers of every nilpotent regular element of $R$ are regular in $R$. Taking $a=\left(\begin{array}{ll}0 & 1 \\ 0 & x\end{array}\right)$ and $u=\left(\begin{array}{ll}0 & 1 \\ 1 & 0\end{array}\right)$, it is easy to see that $a$ is nilpotent and $a u a=a$. But $a^{2}=\left(\begin{array}{ll}0 & x \\ 0 & 0\end{array}\right) \in J(R)$ is not regular. So the condition that all powers of every nilpotent regular element are regular is sufficient but not necessary for strongly $\pi$-regular rings to have stable range one.

\section{REFERENCES}

[1] V.P. Camillo and H.-P. Yu, 'Stable range one for rings with many idempotents', Trans. Amer. Math. Soc. (to appear).

[2] F. Dischinger, 'Sur les anneaux fortement $\pi$-reguliers', C.R. Acad. Sci. Paris, Ser. A 283 (1976), 571-573.

[3] K.R. Goodearl and P. Menal, 'Stable range one for rings with many units', J. Pure Appl. Algebra 54 (1988), 261-287.

[4] P. Menal, 'On $\pi$-regular rings whose primitive factors rings are Artinian', J. Pure Appl. Algebra 20 (1981), 71-81.

[5] L.N. Vaserstein, 'Bass's first stable range condition', J. Pure Appl. Algebra 34 (1984), 319-330.

[6] H.-P. Yu, 'Stable range one for exchange rings', J. Pure Appl. Algebra (to appear).

[7] H.-P. Yu, 'On $\aleph_{0}$-quasi-continuous exchange rings', Comm. Algebra (to appear).

Department of Mathematics

The University of lowa

Iowa City IA 52242

United States of America

e-mail: hpyu@math.uiowa.edu 